\title{
Variable stiffness material based on rigid low-melting-point-alloy- microstructures embedded in soft poly(dimethylsiloxane) (PDMS)
}

\author{
Bryan E. Schubert, ${ }^{* a}$ Dario Floreano $^{a}$
}

Materials with controllable stiffness are of great interest to many fields, including medicine and robotics. In this paper we develop a new type of variable stiffness material based on the combination of a rigid low-melting-point-alloy (LMPA) microstructure embedded in soft poly(dimethylsiloxane) (PDMS). This material can transition between rigid and soft states by controlling the phase of the LMPA through efficient, direct Joule-heating of the LMPA microstructure. The devices tested demonstrate a relative stiffness change of $>25 \times$ (elastic modulus is $40 \mathrm{MPa}$ when LMPA is solid and $1.5 \mathrm{MPa}$ when LMPA is liquid) and a fast transition from rigid to soft states $(<1 \mathrm{~s})$ at low power $(<500 \mathrm{~mW})$. Additionally, the material possesses inherent state (soft and rigid) and strain sensing $(G F=0.8)$ based on resistance changes.

\section{Introduction}

Materials that can controllably change their stiffness are useful in a wide range of fields. Some examples include automotive design, where variable stiffness materials are being created to improve both comfort and safety; ${ }^{1}$ in medicine, variable stiffness materials allow catheters and endoscopes to perform non-invasive diagnostics with greater efficiency and less tissue damage ${ }^{2}$ in aeronautics, new adaptive wing designs for improving flight performance are possible thanks to variable stiffness materials; ${ }^{3,4}$ in civil engineering, materials that can actively change their stiffness are being applied for lowering the damage caused by wind and earthquakes; ${ }^{5}$ in robotics, variable stiffness appendages are able to adapt to different grasping and locomotion tasks. ${ }^{6}$ Examples of controllable stiffness can also be found in nature, such as in the skin of echinoderms (sea cucumbers), ${ }^{7}$ who use changes in dermal stiffness to defend against predators. ${ }^{8}$

Some common engineering materials used to control stiffness make use of smart fluids or shape memory materials. Smart fluids, such as magnetorheological (MR) or electrorheological (ER) fluids, rely on stimulus-induced ordering of nanoor micrometer-sized particles to change their viscosity. In order to use this effect to create controllable stiffness materials, smart fluids must be encapsulated to allow the application of mechanical loads without loss of fluid. When confined, the MR- or ERfluids can be used to change the mechanical properties of the supporting material. Some examples include encapsulation of MR fluids within open-celled foam ${ }^{1}$ or structured elastomeric cavities. ${ }^{9}$ These materials can have fast switching times (ms range), and good relative stiffness changes of $>30 \times$, where the absolute stiffness depends on the structure and mechanical properties of the encapsulation. However, these materials can suffer from high mechanical losses, and in fact, one of their

\footnotetext{
* Corresponding author: bryan.schubert@epfl.ch

${ }^{a}$ Laboratory of Intelligent Systems, Ecole Polytechnique Fédérale de Lausanne, 1015 Lausanne, Switzerland.
}

main applications is in tunable dampers. ${ }^{10}$ Furthermore, the particles can settle out of solution over time and lose functionality. Some of these problems can be circumvented by mixing the MR- and ER-particles directly into solid materials, such as silicone rubber. Unfortunately, this hinders the particles' abilities to align with the applied field, so the relative stiffness changes are greatly reduced $(\sim 0.1 \times) .{ }^{11}$

Shape memory materials, such as shape memory polymer (SMP) and shape memory alloy (SMA), are able to change stiffness through stimulus-induced changes in molecular structure. SMPs have been produced that can change states by exposure to chemical or photo stimuli. ${ }^{12}$ However, the majority of shape memory materials operate through temperature changes, where the material softens as it heats up, undergoing a large change in stiffness once a critical temperature is reached. SMAs have a small relative stiffness change $(<4 \times)$ and a high absolute rest stiffness ( $83 \mathrm{GPa}$ ), whereas SMPs can have a large relative stiffness change $(>100 \times)$ and a wide range of possible rest stiffness $(0.01-3 \mathrm{GPa})$ depending on the material used. ${ }^{13}$ Heat can be applied directly in the case of SMA because it is conductive, but for SMPs heat must be applied indirectly through the use of external heaters. SMPs can be mixed with electrically-conducting particles to remove the need for a separate heater, but the addition of particles increases both the stiffness in the soft and hard states. ${ }^{12,14}$ For thermally-responsive shape memory materials, the stiffness transition speed is related to the thermal conductivity, so SMPs are much slower to heat than SMAs because their thermal conductivity is $\sim 100 \times$ smaller (18 $\mathrm{W} \mathrm{m}^{-1} \mathrm{~K}^{-1}$ for SMAs and 0.15-0.3 $\mathrm{W} \mathrm{m}^{-1} \mathrm{~K}^{-1}$ for SMPs). ${ }^{13}$

In this paper we propose a controllable stiffness material based on the combination of a conductive, phase-changematerial (low-melting-point alloy (LMPA)), and a soft, stretchable encapsulation layer (poly(dimethylsiloxane) (PDMS)), as shown in Figure 1(a). The stiffness of this hybrid material is controlled by direct Joule heating of the LMPA microstructure, which leads to large changes in stiffness as the LMPA mi- 
crostructure melts, while the encapsulating PDMS allows the microstructure to maintain its pre-molten shape in order to preserve its intended electrical and mechanical properties. Figure $1(b, c)$ demonstrates the principle of operation by showing a sample that is able to hold up a metal nut, weighting $10 \times$ more than the sample itself, when the sample is rigid, but then the sample easily bends under the same weight when the microstructure is melted.

Phase change materials are an interesting solution to create variable stiffness materials because they are able to combine the extreme stiffness states of the smart fluid and shape memory material approaches discussed above. This is because phase change materials can completely liquefy, like smart fluids, and completely solidify, like shape memory materials, allowing them to have high relative stiffness changes and low and high absolute stiffness states. Phase change materials have previously been used to create variable stiffness materials; ${ }^{6,15}$ however, the prior solutions make use of external heaters to enable melting, whereas we take advantage of direct Jouleheating of the phase change material (LMPA). This offers a number of advantages such as fast, efficient, direct electrical heating of the LMPA and self-sensing of the shape and physical state of the device (i.e. stiffness). Having this sensory feedback built into the material is a critical innovation for controlling variable stiffness devices in practical applications.

The fabrication method used to create our variable stiffness material consists of filling PDMS microchannels with molten LMPA (see Experimental Section), and it was first developed by Siegel et al. as a generic method for creating arbitrary alloy microstructures in PDMS. ${ }^{16}$ This means that it is suitable for many different types of LMPAs and encapsulating materials, allowing a wide range of customizability in terms of thermal, electrical and mechanical properties. Rather than exploring the whole range of possible combinations, we choose to focus on only one material combination in order to perform an in-depth analysis of some of the fundamental physical principles that govern the material behavior. These principles can then be applied to future systems to precisely engineer the performance needed for a particular application.

The fabrication method also allows the creation of a number of possible microstructures. The particular microstructure that we explore in this paper (see Figure 1(a)) was chosen for its desirable mechanical, electrical and thermal properties. Mechanically, this design provides high bending and stretching stiffness in the length direction. Electrically, the serpentine pattern, typically found in strain gauges, is composed of a long, thin wire that has high resistance, which facilitates the monitoring of thermal and strain effects. Finally, this type of pattern is also typical of resistive heaters, where the close-set, parallel lines serve to concentrate heat, lowering the power and time necessary for melting. Furthermore, the good insulating properties of the PDMS $(\sim 100 \times$ more insulating than the LMPA) limit

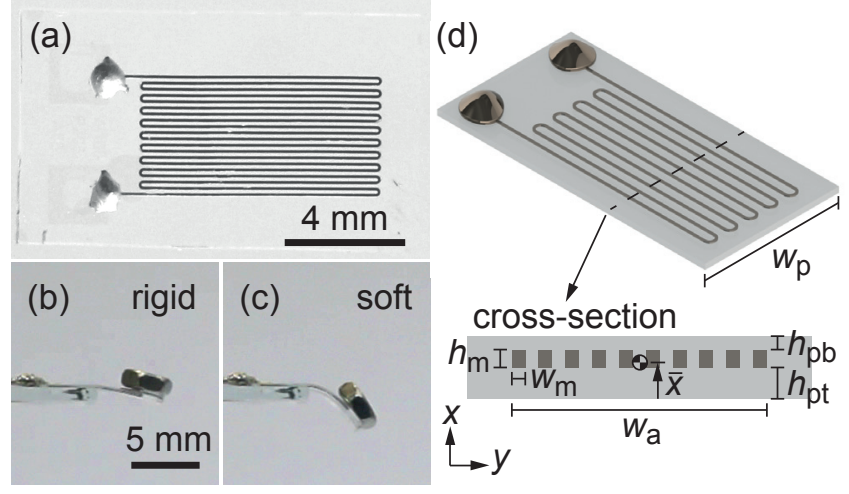

Figure 1 (a) Photograph of a variable stiffness device, showing the low-melting-point alloy (LMPA) tracks embedded in poly(dimethylsiloxane) (PDMS). The LMPA tracks are $100 \mu \mathrm{m}$ wide and $54 \mu \mathrm{m}$ thick, and the total device is $243 \mu \mathrm{m}$ thick. (b) Side view of a device supporting a nut, with $10 \times$ more mass than the variable stiffness device, when the LMPA is solid, and (c) the same device bending under the nut's weight when the LMPA is liquid. (d) Drawing of device showing the cross-sectional dimensions.

thermal loss when heating the LMPA wires. ${ }^{17,18}$ These properties are described in greater detail in the following Results and Discussion Section.

\section{Results and Discussion}

The samples used in the following tests were fabricated to all have the same LMPA $\left(h_{\mathrm{m}}=54 \mu \mathrm{m}\right.$, see Figure $\left.1(\mathrm{~d})\right)$ and PDMS thicknesses $\left(h_{\mathrm{pt}}=79 \mu \mathrm{m}, h_{\mathrm{pb}}=110 \mu \mathrm{m}\right)$, but they have differing line widths $\left(w_{\mathrm{m}}=42,56,106 \mu \mathrm{m}\right)$ and area fraction of alloy $\left(A=N w_{\mathrm{m}} / w_{\mathrm{a}}=0.27,0.28,0.29,0.53,0.80\right.$, where $\mathrm{N}$ is the number of evenly-spaced parallel lines that span $w_{\mathrm{a}}=4$ mm to give the desired $A$ value). Because the LMPA thickness is the same across samples, the increasing area fraction corresponds to a larger volume of LMPA. The samples can be divided into two sets: in one set, the area fraction of LMPA is held constant in order to study the effect of changing the line width $\left(w_{\mathrm{m}} 42-A 0.29, w_{\mathrm{m}} 56-A 0.28, w_{\mathrm{m}} 106-A 0.27\right)$, and in the other set the line width is kept constant so that we can see the effect of changing the LMPA area fraction (i.e. LMPA volume) $\left(w_{\mathrm{m}} 106-A 0.27, w_{\mathrm{m}} 106-A 0.53, w_{\mathrm{m}} 106-A 0.80\right)$.

\subsection{Thermal Behavior}

We first examine the thermal behavior of the LMPA, which is central to the operation of the variable stiffness material. Because the LMPA is both the phase change material and the electrical conductor, we can monitor changes in resistance to determine the state of the alloy, and hence, the softness of the material. This knowledge is critical for controlling the material 
in any practical devices, where operating conditions cannot be known a priori. For instance, contact of the material with random substrates of unknown thermal conductivity will affect the amount of power necessary to melt the LMPA, so this must be compensated for through feedback control.
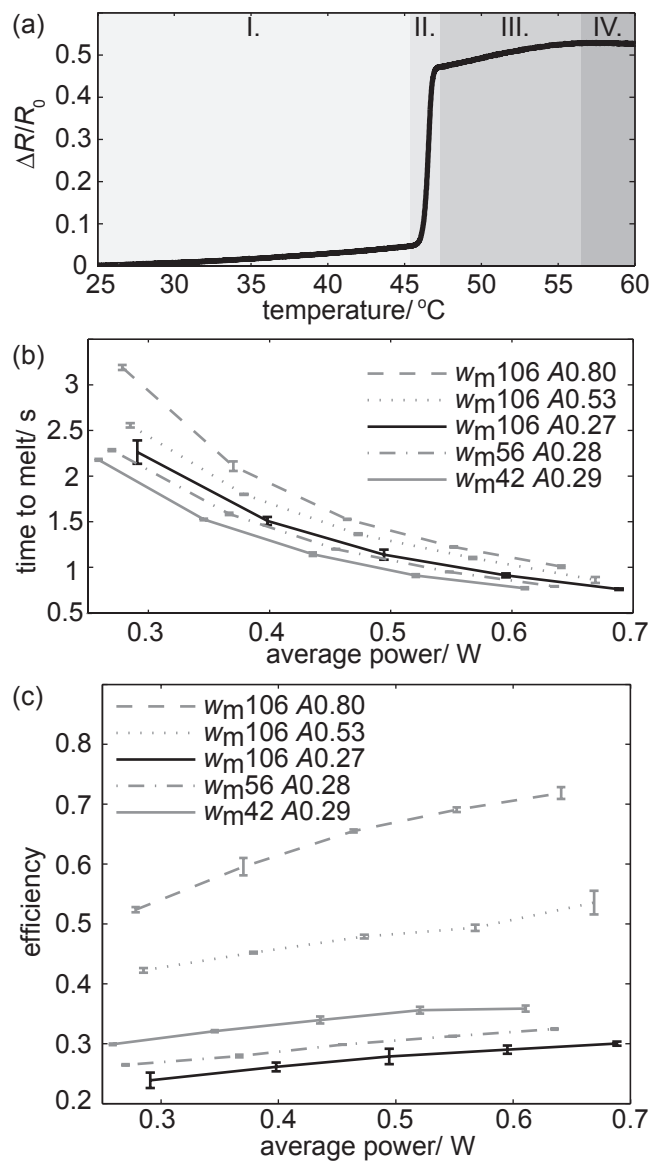

Figure 2 (a) Plot showing the change in resistance of a $w_{\mathrm{m}} 106-A 0.80$ device as it is heated on a hotplate. The shaded regions mark the state of the LMPA: I. heating, II. melting, III. melting of impurities and IV. liquid. (b) Plot of time necessary to melt $90 \%$ of the LMPA by Joule heating at different average electrical powers. (c) Plot of calculated melting efficiency, assuming that the necessary energy to melt $90 \%$ of the LMPA is the energy needed to raise the temperature of all of the LMPA from $25^{\circ} \mathrm{C}$ to 47 ${ }^{\circ} \mathrm{C}$ plus the energy needed to melt $90 \%$ of the LMPA.

\subsubsection{Resistance change with temperature}

Theoretically, the resistance of the LMPA microstructure is related to the standard equation for the resistance of a wire,

$$
R=\rho L / A,
$$

where $\rho$ is the electrical resistivity, $L$ is the length and $A$ is the cross-sectional area. From this equation, we see that the resistance can vary due to changes in geometry or resistivity, both of which can result from temperature changes. Higher temperatures increase electrical resistivity of the LMPA because of increased electron scattering, ${ }^{19}$ and heating also leads to geometric changes that decreases the resistance because, as an isotropic wire undergoes thermal expansion, the cross-sectional area increases faster than the length.

To characterize the change in resistance with temperature we placed a $w_{\mathrm{m}} 106-A 0.80$ sample on a hotplate and slowly increased the temperature. This produced the plot shown in Figure 2(a), where the change in resistance is measured as $\Delta R / R_{0}=\left(R-R_{0}\right) / R_{0}$, with $R=R(T)$ and $R_{0}=R\left(T=25^{\circ} \mathrm{C}\right)$. Figure 2(a) shows that there are four distinct regions of resistance change, and each region has an approximately linear increase that can be expressed as,

$$
\Delta R / R_{0}=\alpha \Delta T
$$

where $\alpha$ a temperature coefficient of electrical resistance that depends on the heating region and $\Delta T$ is the change in temperature. Region I. is due to heating of the solid LMPA, Region II. shows a large change in resistance that is the result of the solid-liquid transition. In region III., the resistance continues to increase due to impurities within the alloy that have a slightly higher melting temperature than the bulk material. Finally, when the whole material is liquid, in region IV., the increase in resistance becomes nearly flat. The change of resistance in each region is primarily due to changes in resistivity of the LMPA because the small temperature change causes limited thermal expansion $\left(\Delta R / R_{0}<-0.01\right.$ due to thermal expansion for $\Delta T=22^{\circ} \mathrm{C}$, see Supplementary Information).

The data shows that it is possible to measure the temperature of a passive device by monitoring the resistance. However, when the material is actively powered to control the stiffness, we can no longer assume that the change in resistance is uniform throughout the device because certain regions will heat up faster than others. In this case, the resistance measured can only be used to estimate the average state of the LMPA (i.e. percent liquid). To estimate the average state of the LMPA we assume that the different parts of the LMPA microstructure are either solid or liquid and we neglect the transitional state in between. Then, using the data from Figure 2(a) we can develop a linear relationship between $0 \%$ LMPA liquid $\left(R<1.05 R_{0}\right)$ and $100 \%$ LMPA liquid $\left(R>1.47 R_{0}\right)$,

$$
\% \text { Liquid }=238\left(\frac{R}{R_{0}}-1.05\right) .
$$

Knowing the percent of the device that is liquid is important for controlling the device stiffness, as will be shown in the Bending Section. 


\subsubsection{Electrically-controlled phase change}

The solid-to-liquid phase change can be controlled through direct Joule heating of the LMPA microstructure. To better understand this method of control, we are interested in knowing the melting speed with respect to the applied electrical power at room temperature $\left(T=25^{\circ} \mathrm{C}\right)$. To perform this measurement, the devices were suspended by their lead wires in still air, and they were supplied a fixed voltage until $\sim 90 \%$ of the LMPA was liquid $\left(R=1.42 R_{0}\right)$. The samples were not allowed to heat until $100 \%$ liquid in order to avoid overheating that can lead to device failure.

The time necessary to melt versus the average supplied power is shown in Figure 2(b). It is possible to melt the devices with less than $100 \mathrm{~mW}$, but this takes more than $10 \mathrm{~s}$, so, for reasons of clarity, only tests of greater than $250 \mathrm{~mW}$ are shown. Figure 2(b) highlights a couple trends related to heating speed. First, having an increasing volume of alloy with a fixed line width, represented by samples $w_{\mathrm{m}} 106-A 0.27, w_{\mathrm{m}} 106-A 0.53$, $w_{\mathrm{m}} 106-A 0.80$, results in slower melting times for the same power. This is not surprising because the energy needed to melt increases with the volume of alloy. Secondly, fixing the volume of alloy and decreasing the line width, represented by samples $w_{\mathrm{m}} 42-A 0.29, w_{\mathrm{m}} 56-A 0.28, w_{\mathrm{m}} 106-A 0.27$, results in faster melting times for the same power. This trend is an effect of improved heating efficiency as shown in Figure 2(c).

The heating efficiency, $\eta$, is estimated by considering the amount of energy needed to melt $90 \%$ of the alloy, $U_{\mathrm{M}}$, divided by the electric energy supplied, $U_{\mathrm{E}}$,

$$
\eta=U_{\mathrm{M}} / U_{\mathrm{E}}=\frac{m\left(c \Delta T+0.9 c_{\mathrm{L}}\right)}{P t},
$$

where $m$ is the mass of alloy, $c=163.176 \mathrm{~J} \mathrm{~kg}^{-1} \mathrm{~K}^{-1}$ is the specific heat of the solid alloy, $c_{\mathrm{L}}=3.682 \times 10^{4} \mathrm{~J} \mathrm{~kg}^{-1}$ is the latent heat of fusion, $T=22{ }^{\circ} \mathrm{C}$ is the change in temperature, $P$ is the electrical power and $t$ is the time to melt (see Figure 2(b)). ${ }^{17}$ Figure 2(c) shows that the efficiency increases as the volume of alloy increases for a fixed line width, and as the line width decreases for a fixed volume of alloy. In both cases, the number of parallel lines increases and the spacing between lines decreases. This improves heat coupling between lines by lowering the volume of PDMS in between lines. Furthermore, the efficiency of all devices improves by supplying higher power because a smaller percentage of the supplied energy is lost to slow conductive and convective cooling through the PDMS.

Cooling of the samples is primarily due to convection to the surrounding air from the PDMS surface. Therefore, the speed of cooling is a function of the final sample temperature and the surface area of the PDMS encapsulation. Since all the experiments heat the samples to approximately the same temperature, and all the samples have the same surface area, the cooling times are all similar. It takes approximately $10 \mathrm{~s}$ for the samples to cool back below melting, but it takes more than $60 \mathrm{~s}$ to cool back to room temperature.

\subsection{Bending}

The bending stiffness is an important metric for defining the forces that the material can support in the thickness direction ( $x$-direction in Figure 3(a)). The theoretical bending stiffness of the device can be estimated for small deflections by considering the Euler beam bending equations. ${ }^{20}$ The stiffness of a cantilever fixed at one end and subjected to a force $F$ at a distance $x=L$ is given by,

$$
k_{\mathrm{b}}=3 E I / L^{3},
$$

where $E$ is the elastic modulus and $I$ is the moment of inertia.

The moment of inertia for our devices is calculated by breaking up the cross-section (see Figure 1(d)) into individual rectangles, each with their own moment of inertia, and then using the parallel axis theorem to find the moment of inertia for the entire beam. ${ }^{20}$ The problem can be further simplified by converting the whole device to one material by re-dimensioning the widths of the rectangles that make up the beam cross-section (see Figure 1(d)) so that they maintain the same bending stiffness when assigned a new elastic modulus. This is done by multiplying each rectangle's width by the ratio of the elastic moduli, $w_{\mathrm{LMPA}}=w_{\mathrm{PDMS}} E_{\mathrm{PDMS}} / E_{\mathrm{LMPA}}\left(E_{\mathrm{PDMS}}=1.81 \mathrm{MPa}\right.$, $E_{\mathrm{LMPA}}=3.00 \mathrm{GPa}$, see Supplementary Information). The calculation of the moment of inertia then proceeds in the standard fashion by first finding the centroid of the cross-section. Because the beam is symmetric in the $y$-direction, the centroid calculation is,

$$
\bar{x}=\frac{\sum_{i} A_{i} x_{i}}{\sum_{i} A_{i}},
$$

where $A_{i}$ is the area of the $i^{\text {th }}$ rectangle and $x_{i}$ is the distance to the center of the rectangle from the bottom of the cantilever. Once the centroid is known, the parallel axis theorem gives the moment of inertia,

$$
I=\sum_{i}\left(I_{i}+A_{i}\left(x_{i}-\bar{x}\right)^{2}\right),
$$

where $I_{i}=w_{i} h_{i}^{3} / 12$ is the moment of inertia for the $i^{\text {th }}$ rectangular section.

When the alloy is liquid, we treat the volume occupied by the alloy as empty space. This is because we assume that the PDMS is incompressible, meaning that the volume of the PDMS channel does not change. Therefore, the LMPA is not subjected to any pressure changes and does not develop any stress.

The results shown in Figure 3(b) follow the trends expected by the model. The samples that all have the same volume of 

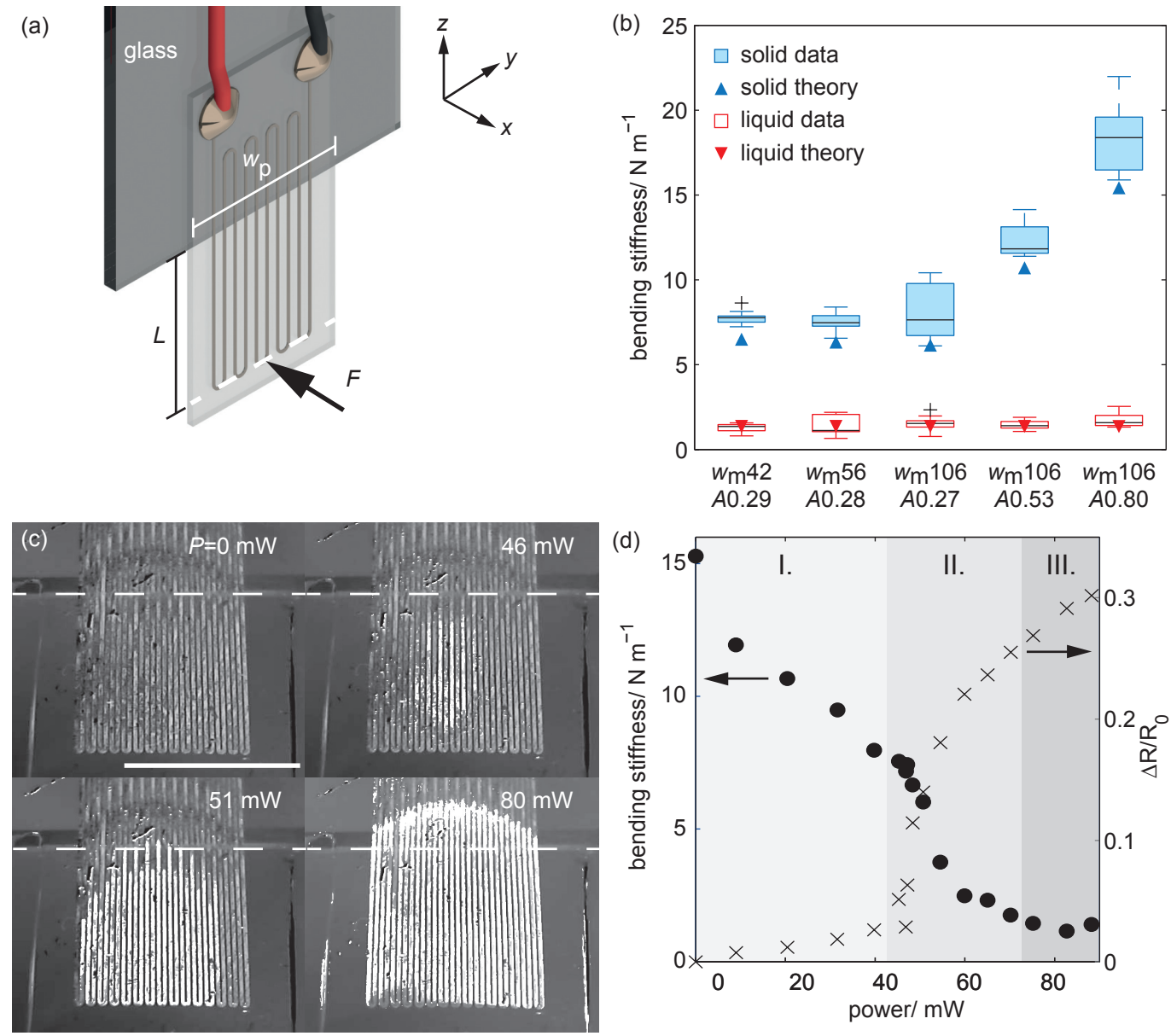

Figure 3 (a) The bending stiffness is measured by fixing the device vertically and applying a deflection at a length, $L=3 \mathrm{~mm}$ (marked by dashed white line), while recording the resulting force $F$. (b) Box plot of the bending stiffness when the LMPA tracks are solid or liquid for the different samples, denoted by their LMPA track widths, $w_{\mathrm{m}}$, and the area fraction of metal, $A$. Three samples of each type were tested three times each while solid and liquid. (c) Images from a $w_{\mathrm{m}} 106-A 0.80$ device as the power is increased. The white areas highlight the portion of the device that is molten. The white areas were generated by image subtraction from the zero-power case. The dashed white line marks the edge of the glass slide. The scale bar is $4 \mathrm{~mm}$. (d) Bending stiffness and change in resistance data collected from the sample shown in (c). The shaded regions mark the state of the LMPA: I. heating, II. melting, and III. liquid past the edge of the glass slide. 
LMPA but differing line widths all show a similar stiffness when solid because the stiffness only depends on the area fraction of the alloy. Furthermore, as the area fraction is increased, the stiffness also increases. Finally, all the samples show a similar stiffness when the LMPA is liquid because the stiffness is dominated by the stiffness of the PDMS encapsulation, which does not vary much between samples because the volume of LMPA is low (4\%-13\%). The model slightly underestimates the bending stiffness of the samples. This is possibly the result of an underestimation of the elastic modulus of the LMPA due to defects in the samples used for modulus testing (see Supplementary Information).

The main discussion so far has been about the two extreme states of the material, but there is also a continuum of stiffness values between the extremes. Figure 3(d) shows the bending stiffness of one $w_{\mathrm{a}} 106-A 0.80$ device as a function of applied power. This plot shows that the material starts to soften as soon as power is supplied. The stiffness behavior can be grouped into three stages. Stage I. is pre-melting, where the gradual stiffness change is a result of thermal softening of the LMPA as it heats up but does not melt, as verified by the low change in resistance. Stage II. is characterized by a sharp drop in stiffness due to melting of the LMPA, as confirmed by the large increase in resistance. Finally, in Stage III., a stable stiffness value is reached once the LMPA has melted past the edge of the glass slide. However, the resistance continues to increase due to further melting of the LMPA, but the increase occurs more gradually because the glass acts as a heat sink. Figure 3(c) further validates the described behavior of the device. Until close to $46 \mathrm{~mW}$, there is no melting observed. After $46 \mathrm{~mW}$, the melting grows radially from the central hot spot until the LMPA is liquid past the edge of the glass support, marked by the dashed white line in Figure 3(c). Besides the change in stiffness of the LMPA, the stiffness of the PDMS is also likely affected by the increase in temperature. Schneider et al. showed that increasing the temperature of PDMS resulted in an increase of the elastic modulus. ${ }^{21}$ This increase depends on the degree of cross-linking, but for PDMS mixed at the standard 10:1 ratio, they observed a linear increase of approximately $10 \%$ in our range of interest $\left(25^{\circ} \mathrm{C}\right.$ to $\left.50^{\circ} \mathrm{C}\right)$. This small change is negligible compared to the much more drastic change in stiffness due to the LMPA melting.

\subsection{Stretching}

The stretching stiffness is a measure of the forces that can be supported by the material in the length direction ( $x$-direction in Figure 4(a)). This stiffness is best characterized by an effective modulus of elasticity. A very simple model for the effective elastic modulus can be derived by assuming that the PDMS and LMPA components act as a single material that undergoes the same strain. In this case, the effective modulus is then related to
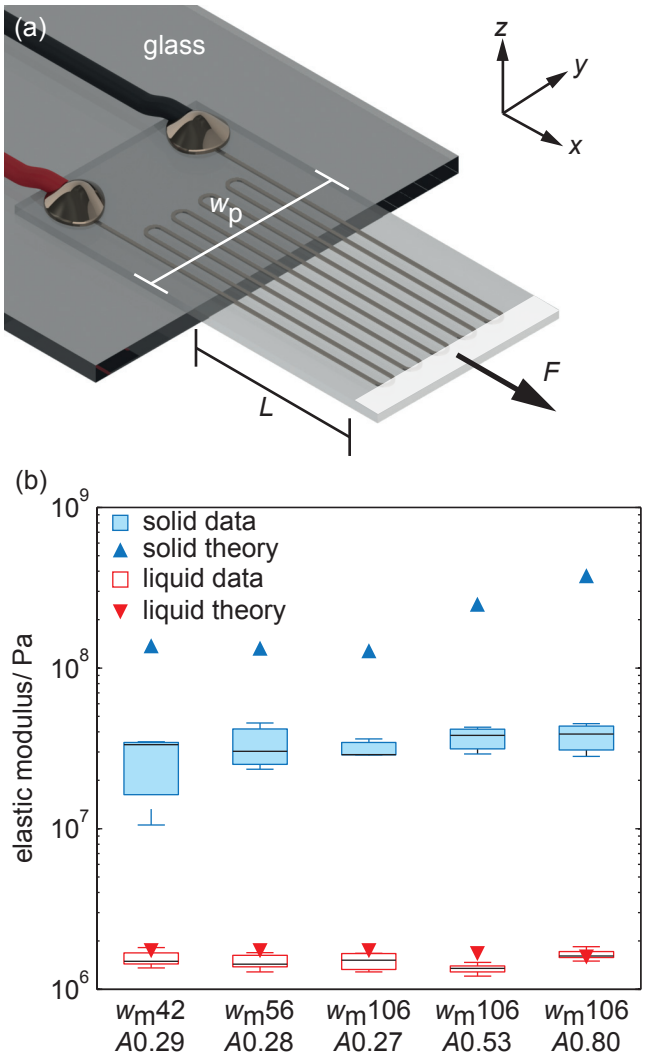

Figure 4 (a) Drawing of stretching tests used to calculate the elastic modulus of the samples. The samples are mounted horizontally and the tip of the samples at length $L$ are clamped between acrylic plates fixed to a force sensor. The clamped region is represented by the white rectangle overlaid on top of the sample. A displacement is applied to the sample, and the resulting force is measured. (b) Box plot of the measured elastic moduli when the LMPA tracks are solid or liquid for different samples, denoted by their LMPA track widths, $w_{\mathrm{m}}$, and the area fraction of metal, $A$. Three samples of each type were each tested three times while liquid and one time while solid because solid testing caused device failure. 
the percent of the cross-sectional area taken by each material,

$$
E_{\mathrm{solid}}=\left(A_{\mathrm{LMPA}} E_{\mathrm{LMPA}}+A_{\mathrm{PDMS}} E_{\mathrm{PDMS}}\right) / A \text {. }
$$

For the case where the alloy is fully liquid, we again assume incompressibility of PDMS so that there is no change in the PDMS channel volume and the liquid LMPA develops no stress. Therefore, the expression simplifies to $E_{\text {liquid }}=$ $A_{\mathrm{PDMS}} E_{\mathrm{PDMS}} / A$.

The effective elastic modulus is measured for all the samples by stretching the material in both solid and liquid states (see Figure 4(b)). The experiment can be performed multiple times when the LMPA is liquid. However, it can only be performed once when the LMPA is solid because the stretching breaks the samples.

The results show that the theory proposed closely predicts the liquid phase modulus, but it greatly overestimates the solid phase modulus. This can be explained by observations that portions of the PDMS encapsulation detach from the LMPA microstructure when strained. This means that the two materials do not experience the same strain, as the model assumes. The softer PDMS portion of the material therefore lowers the observed stiffness, and in fact obscures any trends that would result from having a higher volume of LMPA. This is why all the solid phase measurements have nearly the same moduli. Finite element modeling would be required to get a more accurate prediction of the stiffness. However, this simple model is still useful for estimating the liquid phase modulus and for quickly establishing an upper bound on the solid phase modulus.

\subsection{Strain Sensing}

The controllable stiffness device can function as a strain sensor in both liquid and solid states, which would be useful in applications where knowledge of the shape and deformation of the device is important, such as in a robotic appendage.

In both liquid and solid states, the change in resistance with bending is very low for this design because the alloy tracks are near the neutral axis of bending, and therefore do not experience a significant change in geometry. The resistance change with stretching is higher, but, for the solid case, this results in plastic deformation or breaking of the LMPA. The plastic deformation can be easily fixed by re-melting the alloy, but, as will be shown in the Failure Modes Section, complete breaks cannot always be easily fixed. However, if the LMPA is liquid, reliable and reversible, resistive strain sensing is possible, and is similar to current soft strain sensors composed of eutectic metal alloys that are liquid at room temperature. ${ }^{22-24}$

To model the sensing behavior, we consider the PDMS as an incompressible, linear-elastic solid, and we make a small strain assumption about the Poisson effect. We also assume that the resistance follows the relationship given previously, $R=$ $\rho L / A$, but we consider the resistivity to be constant. Then, we can estimate the theoretical gauge factor $\left(G F=\left(\Delta R / R_{0}\right) / \varepsilon\right)$ for this device as,

$$
G F=(2-0.25 \varepsilon) /(1-0.5 \varepsilon)^{2},
$$

where $\varepsilon$ is the strain. This neglects the contribution of the curved wire ends because they represent a small portion of the sample. Also, in the experiments described below, the ends of the wires are clamped, so they do not experience strain.
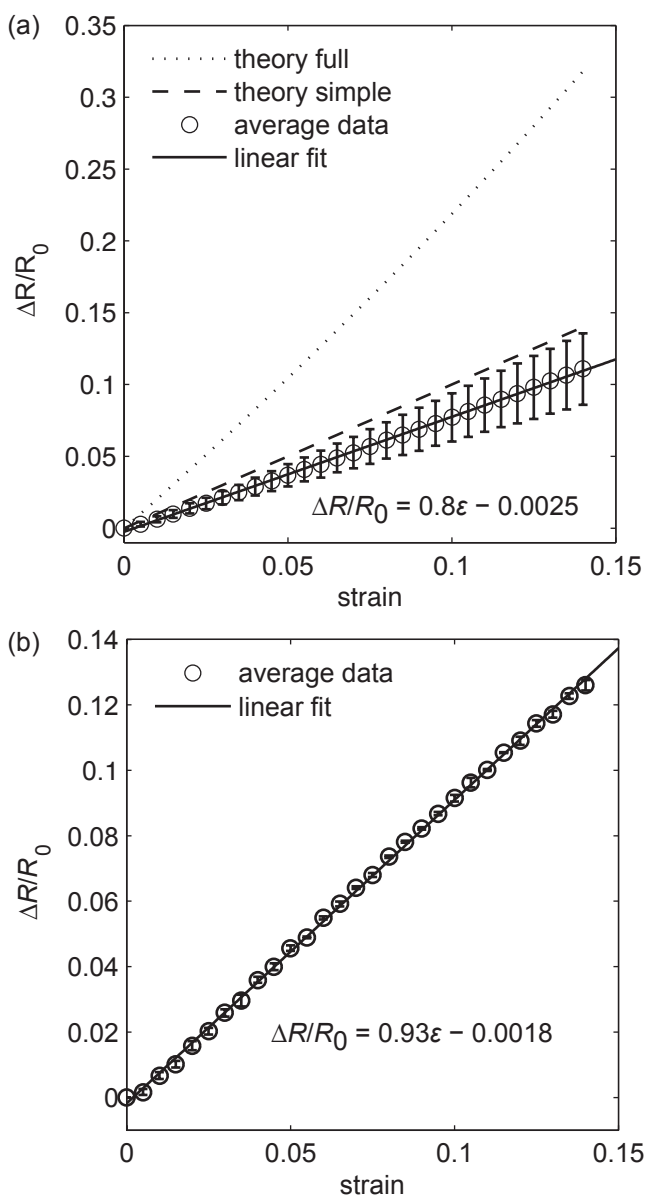

Figure 5 (a) Plot of average change of resistance versus strain from stretching (see Figure 4(a)) while the LMPA was liquid. Three samples of each type $\left(w_{\mathrm{m}} 106-A 0.27, w_{\mathrm{m}} 106-A 0.53\right.$, $\left.w_{\mathrm{m}} 106-A 0.80, w_{\mathrm{m}} 56-A 0.28, w_{\mathrm{m}} 42-A 0.29\right)$ were tested three times each. The simple theory line represents a change of resistance equal to the strain $(G F=1)$, and the full theory assumes a change in resistance from both strain and the Poisson effect, Equation 9. (b) Plot of the average change in resistance with strain for a $w_{\mathrm{m}} 106-A 0.27$ sample tested three times in a row.

To test the strain sensing properties of the devices, we recorded the change in resistance as we stretched the samples at a fixed speed (see Figure 4(a)). There were no apparent trends 
related to line width or the area fraction of alloy, so we grouped all the data into an average line shown in Figure 5(a).

The measured $G F$ is smaller than the predicted value, and it appears to follow a linear trend. Chossat et al. noticed similar behavior in their eutectic Gallium Indium sensor. ${ }^{22}$ Their sensor very nearly followed a relationship where the change in resistance was equal to the strain, giving them a $G F=1$. They argued that this was possibly due to the high surface tension of the alloys oxidized skin inhibiting the alloy's ability to conform to the changing shape of the channel. It is likely that our alloy experiences a similar circumstance, leading to our lower than expected average $G F$ of 0.8 . The fairly high standard deviation may be due to variations in filling of the microstructure. Incomplete filling caused by the introduction of trapped air can have a large affect on device performance. However, individual sensors show very little change in $G F$ between repeated stretching trials, as shown in Figure 5 (b), where the change in resistance with strain of a $w_{\mathrm{m}} 106-A 0.27$ sample is averaged over three sequential trials. The standard deviation for these three trials is very small. Therefore, while it may not be possible to develop a model for the GF a priori due to the high standard deviation between devices, calibration of individual devices should yield very repeatable results.

\subsection{Failure Modes}

For practical use, it is important to know the limits of the material before failure. Our material has two main failure mechanisms, depending on the state of the structure. When the alloy is solid, the main source of failure comes from the LMPA tracks breaking when stretched. For all the samples tested (five different types and three of each type), the average strain at break was $3.2 \%$, with some samples breaking with as little as $0.2 \%$ strain and some surviving up to $9.8 \%$ strain. There was no observed trend related to the different geometries tested. In contrast, the tests used to determine the elastic modulus of solid LMPA (see Supplementary Information) had an average of $9.4 \%$ strain at break. The reason for the lower strain limit of the LMPA in the microstructures is likely a result of stress concentrations, due to non-uniform stretching of the PDMS encapsulation, and defects in the alloy tracks.

Solid breaks can occasionally be repaired when the applied strain is removed. If the break is clean, then relaxing the material puts the two halves of the break back in contact, restoring the conductivity of the device. Then, Joule heating can be used to re-melt the contact and restore continuity of the LMPA wire, as shown in Figure 6(a).
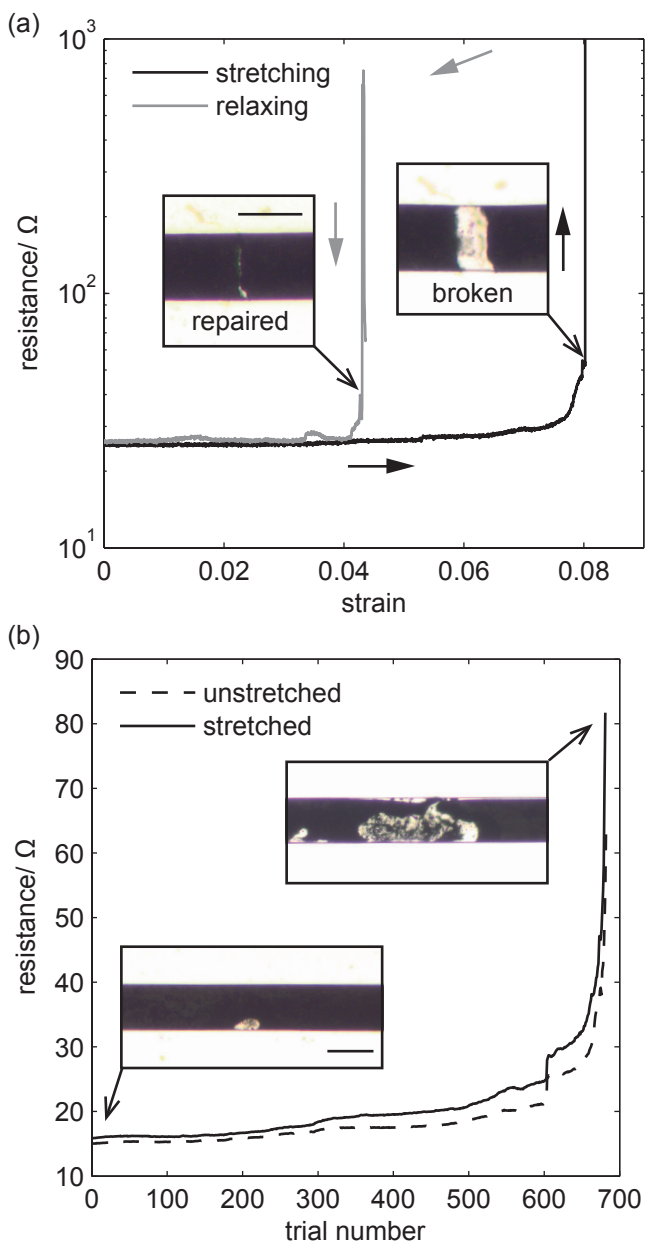

Figure 6 (a) Resistance versus strain for a $w_{\mathrm{m}} 106-A 0.80$ sample stretched while the LMPA was solid. As the strain is increased, the LMPA eventually breaks and the resistance goes to infinity. Then, when the strain is released, the broken LMPA tracks come back into contact, restoring the resistance. The inset pictures show how this looks on a different $w_{\mathrm{m}} 106-A 0.27$ sample. (b) Stretched (10\% strain) and unstretched ( $0 \%$ strain) resistance of a $w_{\mathrm{m}} 106-A 0.27$ sample with liquid LMPA over hundreds of stretching cycles. The insets highlight one possible failure mode resulting from bubble growth as the LMPA degrades due to overheating of the defective area. The scale bars on the insets are $100 \mu \mathrm{m}$.

Although the solid LMPA microstructure is susceptible to failure in stretching, in pure bending, the solid LMPA tracks can go through large deflections without breaking. We have observed samples still working even after being folded $180^{\circ}$ from flat. The LMPA tracks have a large resistance to bending failures because they are thin structures located near the neutral axis, and therefore, they do not experience high strain under bending.

When the LMPA is liquid, we have observed the material supporting strains near $30 \%$ without loss of resistance. How- 
ever, repeated stretching can lead to failure stemming from bubble formation. Figure 6(b) shows the resistance of a $w_{\mathrm{m}} 106-$ $A 0.27$ sample unstretched and stretched to $10 \%$ strain over hundreds of cycles. The resistance gradually increases until rapid deterioration after 600 stretches. The inset pictures show a bubble before stretching and one after stretching repeatedly. The region where the bubble is located has a narrower cross section than the adjacent areas. This can lead to local overheating and degradation of the material that is propagated by repeated stretching until failure.

Failure of the LMPA does not mean the end of use for the whole device. If conductivity is completely lost, fresh LMPA can easily be re-flowed through the microchannels to restore proper functioning. The devices used in this paper were repaired several times after breaking, and they showed only slight variations in properties. Mainly, the difference was in the base resistance due to non-uniform filling. Therefore, measuring the resistance after fabrication can give an indication of the quality of the LMPA track, where lower resistances are related to better filling.

\section{Conclusion}

We have developed a new type of smart, variable stiffness material. The devices presented here demonstrate a good relative stiffness change of $>25 \times$ (elastic modulus of $40 \mathrm{MPa}$ when LMPA is solid and 1.5 MPa when LMPA is liquid), a fast transition from rigid to soft states $(<1 \mathrm{~s})$ at low power $(<500 \mathrm{~mW})$, and inherent thermal and strain sensing $(G F=0.8)$. However, the measured values are not representative of the limits of this technology because there exists a high level of possible customization of the materials and design. Much higher relative stiffness changes $(>1000 \times)$ are possible simply by increasing the proportion of LMPA that makes up the total volume of the device (the tested devices only had 4-13\% LMPA by volume). Furthermore, different absolute stiffness values for the rigid and soft states can be achieved by changing the materials and the alloy microstructure design. Even more functionality could be added by using an encapsulation material that also softens with temperature, such as SMP. This would result in larger relative stiffness changes and stiffness range, as well as, the possibility of having multiple transition regions. In terms of strain sensing, careful LMPA microstructure design could increase strain sensitivity and allow sensing of multiple strain directions. Finally, the material can be scaled up to the size of a wafer by using the current fabrication techniques, or even larger structures are possible by using standard molding processes. The relative stiffness will not change as a particular design is scaled, however, having higher volumes of LMPA and PDMS will increase the power requirements and decrease the melting speeds. Therefore, when scaling it may be important to consider alternative designs that minimize power requirements and melting time while maximizing stiffness, such as three-dimensional scaffolds that minimize metal volume. ${ }^{25}$

\section{Experimental}

Device fabrication: Samples were fabricated using the technique originally outlined by Siegel et al. ${ }^{16}$ Microchannel molds were fabricated by photolithography of SU-8 (GM 1070, Gersteltec) on silicon. The developed molds were exposed to chlorotrimethylsilane (Sigma-Aldrich Company Ltd.) in vacuum for one hour to lower adhesion. Then, spin-coating was used to apply PDMS (Sylgard 184, Dow Corning) to the mold wafers and to blank wafers to form the top and bottom of the device, respectively. After curing at $80{ }^{\circ} \mathrm{C}$ for 2 hours, the patterned PDMS was peeled from the mold. Next, inlet and outlet holes were punched in the microchannel layer and both layers were exposed to oxygen plasma for 30 seconds before being bonded by pressing them together. We skipped the silanization of the channels used by Siegel et al. because initial tests showed no advantage in terms of filling over untreated channels. The microchannels were filled with LMPA (Cerrolow 117, HiTech Alloys; composition by weight: $45 \%$ bismuth, $23 \%$ lead, $19 \%$ indium, $8 \%$ tin, $5 \%$ cadmium; $47^{\circ} \mathrm{C}$ melting temperature). The empty PDMS encapsulation was placed on a hotplate at $80{ }^{\circ} \mathrm{C}$ and a small droplet of LMPA was melted over the inlet hole. Then, a syringe was used to apply positive pressure to the alloy to force it through the channels. After filling, additional LMPA was melted over the inlet and outlet holes and copper wires were attached. Then, the whole device was allowed to cool to room temperature. The width of the samples was trimmed by hand with a razor blade, and the final dimensions of the samples were $w_{\mathrm{p}}=5.7 \pm 0.2 \mathrm{~mm}$, $w_{\mathrm{a}}=4.0 \pm 0.05 \mathrm{~mm}, h_{\mathrm{pt}}=79 \pm 4 \mu \mathrm{m}, h_{\mathrm{m}}=54 \pm 4 \mu \mathrm{m}$ and $h_{\mathrm{pb}}=110 \pm 3 \mu \mathrm{m}$ (see Figure 1(d)) as measured by optical microscope (Olympus IX73; $20 \times$ PlanC N objective). The line widths were $w_{\mathrm{m}}=42,56,106 \pm 1 \mu \mathrm{m}$, and the area fractions of alloy for the particular line widths were $w_{\mathrm{m}} 42: A=0.29 \pm 0.01$; $w_{\mathrm{m}} 56: A=0.28 \pm 0.01 ; w_{\mathrm{m}} 106: A=0.27,0.53,0.80 \pm 0.01$.

Electrical measurements: Resistance and power of the devices were measured using LabVIEW (National Instruments). To determine the resistance of the devices, we used a voltage divider circuit composed of a known reference resistor $\left(R_{\mathrm{r}}=14.8\right.$ $\Omega$ ) in series with the device under test (DUT). A fixed voltage was supplied by a DC power supply (M10-DP-305E, Shanghai MCP Corp.). By measuring the voltage across the reference resistor, $V_{\mathrm{r}}$, we could determine the current through the circuit, $I$. Then, dividing the voltage measured across the DUT, $V_{\mathrm{DUT}}$, by the current gave the DUT resistance, $R_{\mathrm{DUT}}=V_{\mathrm{DUT}} / I$. The electrical power was calculated as $P_{\mathrm{DUT}}=V_{\mathrm{DUT}} I$.

Thermal measurements: The characterization of resistance 
with temperature was performed using a $w_{\mathrm{m}} 106-A 0.80$ sample placed in an insulated box on a hotplate (C-MAG HS 7, IKA). The resistance was measured using the voltage divider already mentioned, and temperature was recorded using a temperature probe (AD22103KT, Analog Devices) attached to the surface of the sample. The temperature was increased at a rate of approximately $0.01{ }^{\circ} \mathrm{C} \mathrm{s}^{-1}$.

For melting speed tests, one sample of each size was suspended by its lead wires in still air $\left(25^{\circ} \mathrm{C}\right)$. Then, a fixed voltage was suddenly applied across the device, and the resistance and power were measured as the device heated. When the resistance reached $1.42 \times R_{0}$, representing $90 \%$ liquid (see Thermal Behavior Section), the voltage was switched off, and the device was allowed to cool back to room temperature. This was repeated three times for each sample.

Bending and stretching tests: For bending and stretching tests, the half of the device containing the inlet and outlet holes was oxygen-plasma bonded to a glass microscope slide to provide a rigid support to hold the sample during testing (see Figures 3(a) and 4(a)). Solid and liquid tests were performed in air at $25^{\circ} \mathrm{C}$. For liquid tests, the LMPA was supplied sufficient power to melt the LMPA microstructure past the edge of the glass slide (between $70-150 \mathrm{~mW}$ ), and bending or stretching was only initiated after the device had reached a stable equilibrium. Between solid bending tests, the LMPA was melted to remove any effects of plastic deformation of the LMPA.

In bending tests, the samples were mounted perpendicular to the ground (see Figure 3(a)), and their free ends were deflected in the $-x$-direction by a one-axis load cell (UF1, Applied Measurement Limited; $540 \mathrm{mN}$ range, $79 \mu \mathrm{N}$ ) mounted to a linear stage (T-LSR150B-KT03, Zaber; $0.496 \mu \mathrm{m}$ microstep resolution, $<2.5 \mu \mathrm{m}$ repeatability, $150 \mathrm{~mm}$ range). A $200 \mu \mathrm{m}$ thick strip of polyester was attached to the end of the load cell to apply the force on the sample across a line $3 \mathrm{~mm}$ distance from the glass edge (see Figure 3(a)). The total deflection was 300 $\mu \mathrm{m}$ at a speed of $100 \mu \mathrm{m} \mathrm{s}^{-1}$.

In stretching tests, the samples were mounted parallel to the ground (see Figure 4(a)), and their free ends were clamped between pieces of acrylic that were attached between a six-axis force/torque sensor (Nano 17 SI-12-0.12, ATI Industrial Automation; only used $z$-axis: $17 \mathrm{~N}$ range, $1 \mathrm{mN}$ resolution) and the same linear stage already mentioned. Displacements representing $10-20 \%$ strain were performed at $100 \mu \mathrm{m} \mathrm{s}^{-1}$. Elastic modulus values were extracted from the linear portion of the stress strain data (1\% strain for samples with liquid LMPA and $0.5 \%$ strain for solid LMPA).

\section{Acknowledgements}

This work was supported by the Swiss National Science Foundation through the National Center for Competence in Research
(NCCR) Robotics. The authors thank Jürg Germann, Pavan Ramdya and Stefano Varricchio for their helpful input on the manuscript.

\section{References}

[1] S. S. Deshmukh, PhD thesis, Massachussetts Institute of Technology, 2007.

[2] A. Loeve, P. Breedveld and J. Dankelman, IEEE Pulse, 2010, 1, 26-41.

[3] A. Y. N. Sofla, S. A. Meguid, K. T. Tan and W. K. Yeo, Mater. Des., 2010, 31, 1284-1292.

[4] C. Thill, J. Etches, I. Bond, K. Potter and P. Weaver, The Aeronautical Journal, 2008, 112, 117-139.

[5] L. Janke, C. Czaderski, M. Motavalli and J. Ruth, Mater. and Struct., 2005, 38, 578-592.

[6] H. Nakai, Y. Kuniyoshi, M. Inaba and H. Inoue, Proc. 2002 IEEE/RSJ Intl. Conf. Intelligent Robots and Systems, 2002, 2025-2030.

[7] I. C. Wilkie, J. Exp. Bio., 2002, 205, 159-165.

[8] J. R. Capadona, K. Shanmuganathan, D. J. Tyler, S. J. Rowan and C. Weder, Science, 2008, 319, 1370-1374

[9] C. Majidi and R. J. Wood, Appl. Phys. Lett., 2010, 97, 164104.

[10] G. Z. Yao, F. F. Yap, G. Chen, W. H. Li and S. H. Yeo, Mechatronics, 2002, 12, 963-973.

[11] M. Kallio, T. Lindroos, S. Aalto, E. Järvinen, T. Kärnä and T. Meinander, Smart Mater. Struct., 2007, 16, 506-514.

[12] M. Behl and A. Lendlein, Mater. Today, 2007, 10, $20-28$.

[13] C. Liu, H. Qin and P. T. Mather, J. Mater. Chem., 2007, 17, 1543-1558.

[14] P. T. Mather, X. Luo and I. A. Rousseau, Annu. Rev. Mater. Res., 2009, 39, 445-471.

[15] W. Shan, T. Lu and C. Majidi, Smart Mater. Struct., 2013, 22, 085005.

[16] A. C. Siegel, D. A. Bruzewicz, D. B. Weibel and G. M. Whitesides, Adv. Mater., 2007, 19, 727-733.

[17] The Bendalloy Inc., Physical properties sheet Lens 117 alloy, http://www.bendalloy.co.uk/page7.html, 2012.

[18] Dow Corning Corp., Sylgard 184 silicone elastomer, data sheet Ref. No. 10-1204A-01, http://www.dowcorning.com, 1998-1999.

[19] B. M. Smirnov, Principles of Statistical Physics, Wiley-VCH Verlad GmbH \& Co. KGaA, Weinham, Germany, 2006.

[20] E. P. Popov, Engineering Mechanics of Solids, Prentice-Hall, Inc., New Jersey, USA, 2nd edn, 1998.

[21] F. Schneider, T. Fellner, J. Wilde and U. Wallrabe, J. Micromech. Microeng., 2008, 18, 065008.

[22] J.-B. Chossat, Y.-L. Park, R. J. Wood and V. Duchaine, IEEE Sens. J., 2013, 1-5.

[23] Y.-L. Park, B.-R. Chen and R. J. Wood, IEEE Sens. J., 2012, 12, 27112718. 
[24] R. D. P. Wong, J. D. Posner and V. J. Santos, Sens. Actuators, A, 2012, [25] T. A. Schaedler, A. J. Jacobsen, A. Torrents, A. E. Sorensen, J. Lian, J. R. 179, 62-69. Greer, L. Valdevit and W. B. Carter, Science, 2011, 334, 962-965. 